University of Nebraska - Lincoln

DigitalCommons@University of Nebraska - Lincoln

4-28-2008

\title{
Polarization switching kinetics at the nanoscale in ferroelectric copolymer Langmuir-Blodgett films
}

\author{
R. V. Gaynutdinov \\ Institute of Crystallography, Russian Academy of Sciences, 119333 Moscow, Russia \\ O. A. Lysova \\ Institute of Crystallography, Russian Academy of Sciences, 119333 Moscow, Russia
}

A. L. Tolstikhina

Institute of Crystallography, Russian Academy of Sciences, 119333 Moscow, Russia

S. G. Yudin

Institute of Crystallography, Russian Academy of Sciences, 119333 Moscow, Russia

V. M. Fridkin

Institute of Crystallography, Russian Academy of Sciences, 119333 Moscow, Russia

See next page for additional authors

Follow this and additional works at: https://digitalcommons.unl.edu/physicsducharme

Part of the Physics Commons

Gaynutdinov, R. V.; Lysova, O. A.; Tolstikhina, A. L.; Yudin, S. G.; Fridkin, V. M.; and Ducharme, Stephen, "Polarization switching kinetics at the nanoscale in ferroelectric copolymer Langmuir-Blodgett films" (2008). Stephen Ducharme Publications. 39.

https://digitalcommons.unl.edu/physicsducharme/39

This Article is brought to you for free and open access by the Research Papers in Physics and Astronomy at DigitalCommons@University of Nebraska - Lincoln. It has been accepted for inclusion in Stephen Ducharme Publications by an authorized administrator of DigitalCommons@University of Nebraska - Lincoln. 


\section{Authors}

R. V. Gaynutdinov, O. A. Lysova, A. L. Tolstikhina, S. G. Yudin, V. M. Fridkin, and Stephen Ducharme 


\title{
Polarization switching kinetics at the nanoscale in ferroelectric copolymer Langmuir-Blodgett films
}

\author{
R. V. Gaynutdinov, ${ }^{1}$ O. A. Lysova, ${ }^{1}$ A. L. Tolstikhina, ${ }^{1}$ S. G. Yudin, ${ }^{1}$ V. M. Fridkin,,${ }^{1,2}$ and \\ Stephen Ducharme ${ }^{2, a)}$ \\ ${ }^{1}$ Institute of Crystallography, Russian Academy of Sciences, 119333 Moscow, Russia \\ ${ }^{2}$ Department of Physics and Astronomy, Nebraska Center for Materials and Nanoscience, \\ University of Nebraska, Lincoln, 68588-0111, Nebraska, USA
}

(Received 26 February 2008; accepted 4 April 2008; published online 28 April 2008)

\begin{abstract}
The polarization switching kinetics of ferroelectric Langmuir-Blodgett films of vinylidene fluoride-trifluoroethylene copolymer were investigated by piezoresponse force microscopy with a resolution of $100 \mathrm{~nm}$. The switching time in response to a localized voltage pulse exhibits an exponential dependence on reciprocal voltage, which is consistent with nucleation-limited switching dynamics. () 2008 American Institute of Physics. [DOI: 10.1063/1.2916707]
\end{abstract}

There is increasing interest in the dynamics of polarization at the nanoscale in ferroelectric thin films motivated by the application of ferroelectric thin films to, e.g., nonvolatile random-access memories, ${ }^{1}$ and enabled by the advances in the technology of ferroelectric thin film fabrication and nanoscale patterning and the application of scanning probe microscopy to probe ferroelectric properties at the nanoscale. ${ }^{2}$ Piezoelectric force microscopy (PFM) has become the preferred method for imaging polarization domain structure $^{3}$ and dynamics ${ }^{4}$ in ferroelectric films. Studies of ferroelectricity in films of nanometer thickness were made with Langmuir-Blodgett (LB) films of copolymers of vinylidene fluoride and trifluoroethylene copolymers $\mathrm{P}(\mathrm{VDF}-\mathrm{TrFE}){ }^{5,6}$ Although the equilibrium ferroelectric properties of the LB films are weakly dependent on the thickness, ${ }^{7}$ the polarization switching properties are sensitive to both film thickness ${ }^{8,9}$ and LB fabrication conditions, ${ }^{10,11}$ indicating that crystal morphology plays an essential role. These switching studies, however, were all made with largearea (of order $1 \mathrm{~mm}^{2}$ ) polycrystalline LB film capacitors and, therefore, could not reveal detailed domain structures nor discern the role of crystal morphology. Nanoscale piezoelectric imaging studies of $\mathrm{P}(\mathrm{VDF}-\mathrm{TrFE})$ films made by solvent spinning ${ }^{12}$ and LB deposition ${ }^{13}$ indicated a connection between domain structure and crystal morphology. Recent PFM studies ${ }^{14}$ with an imaging resolution of $5 \mathrm{~nm}$ have made this clear by demonstrating that the polarization of LB copolymer films could be patterned with feature sizes as small as the crystallites, which is $30-50 \mathrm{~nm}$. Here, we report a PFM study of polarization switching kinetics, probing a region approximately $500 \mathrm{~nm}$ in diameter in ferroelectric copolymer LB films.

The ferroelectric random copolymer consisting of $70 \%$ vinylidene fluoride and 30\% trifluoroethylene (70:30 copolymer) was deposited to the desired thickness by horizontal LB deposition, as described in detail elsewhere. ${ }^{15}$ Briefly, in this method, a solution of $1.3 \%$ by weight copolymer in dimethyl-sulfoxide was dispersed onto the surface of a subphase of ultrapure water at room temperature. Barriers on the water's surface slowly compressed the Langmuir film to a surface pressure of $3 \mathrm{mN} / \mathrm{m}^{2}$, which is well below the

\footnotetext{
${ }^{a)}$ Electronic mail: sducharme1@unl.edu.
}

collapse pressure. The copolymer LB films were transferred layer by layer to a silicon substrate coated with $50 \mathrm{~nm}$ thick aluminum film. The samples consisting of 10,30 and 120 ML were then annealed at $120^{\circ} \mathrm{C}$ for $3 \mathrm{~h}$ and cooled to room temperature to optimize the crystallinity and stabilize the ferroelectric properties. The LB film fabrication methods are described in detail elsewhere. ${ }^{5,15}$ The film thickness is estimated to be $1.8 \mathrm{~nm}$ per $1 \mathrm{ML}$ transfer, based on spectroscopic ellipsometery studies made on similarly prepared LB films of this copolymer. ${ }^{16}$ Measurements were carried out with scanning probe microscope (model Solver P47 from NT-MDT, Moscow) operating in contact mode for imaging both topography [atomic force microscopy (AFM)] and piezoresponse (PFM). The measurements were made with silicon cantilevers (model CSC38 from MicroMasch, Estonia), which had a lever force constant of approximately $0.05 \mathrm{~N} / \mathrm{m}$, a resonant frequency of $21 \mathrm{kHz}$, and a tip radius of $10 \mathrm{~nm}$ or less. The PFM measurements were made by applying an excitation voltage of amplitude $1.5 \mathrm{~V}$ and frequency $200 \mathrm{kHz}$ to the tip and recording the resulting tip deflection signal from the position detector with a lock-in amplifier. The measurements were carried out in air in a class 100000 clean room maintained at temperature of $26 \pm 0.05{ }^{\circ} \mathrm{C}$ and relative humidity of $40 \pm 1 \%$. For the PFM measurements, a CSC38 tip was coated with Ti/Pt conductive coating and had an estimated radius of $40 \mathrm{~nm}$ and an estimated imaging resolution of $60 \mathrm{~nm}$, which is insufficient to resolve individual crystal grains, which are typically $30 \mathrm{~nm}$ in size in the copolymer LB films. ${ }^{14}$

The local polarization switching studies were conducted in two operating regimes, which are switching spectroscopy for measurement of the local hysteresis loop and voltage pulse switching followed by PFM for imaging of the switched spot. In switching spectroscopy, the piezoresponse was recorded after each of a series of voltage pulses cycling between positive and negative limits. ${ }^{4}$ The local piezoresponse hysteresis loops of representative spots on the three films are shown in Fig. 1. The hysteresis loop from the 10 ML film [Fig. 1(a)] exhibits a coercive voltage of $V_{c}$ $=2.3 \mathrm{~V}$, defined as the average voltage amplitude at which the piezoresponse crosses zero. The loop does not appear to be saturated, meaning that this is a lower limit of the coercive field. The hysteresis loop from the $30 \mathrm{ML}$ film [Fig. 1(b)] appears well saturated with a coercive voltage 

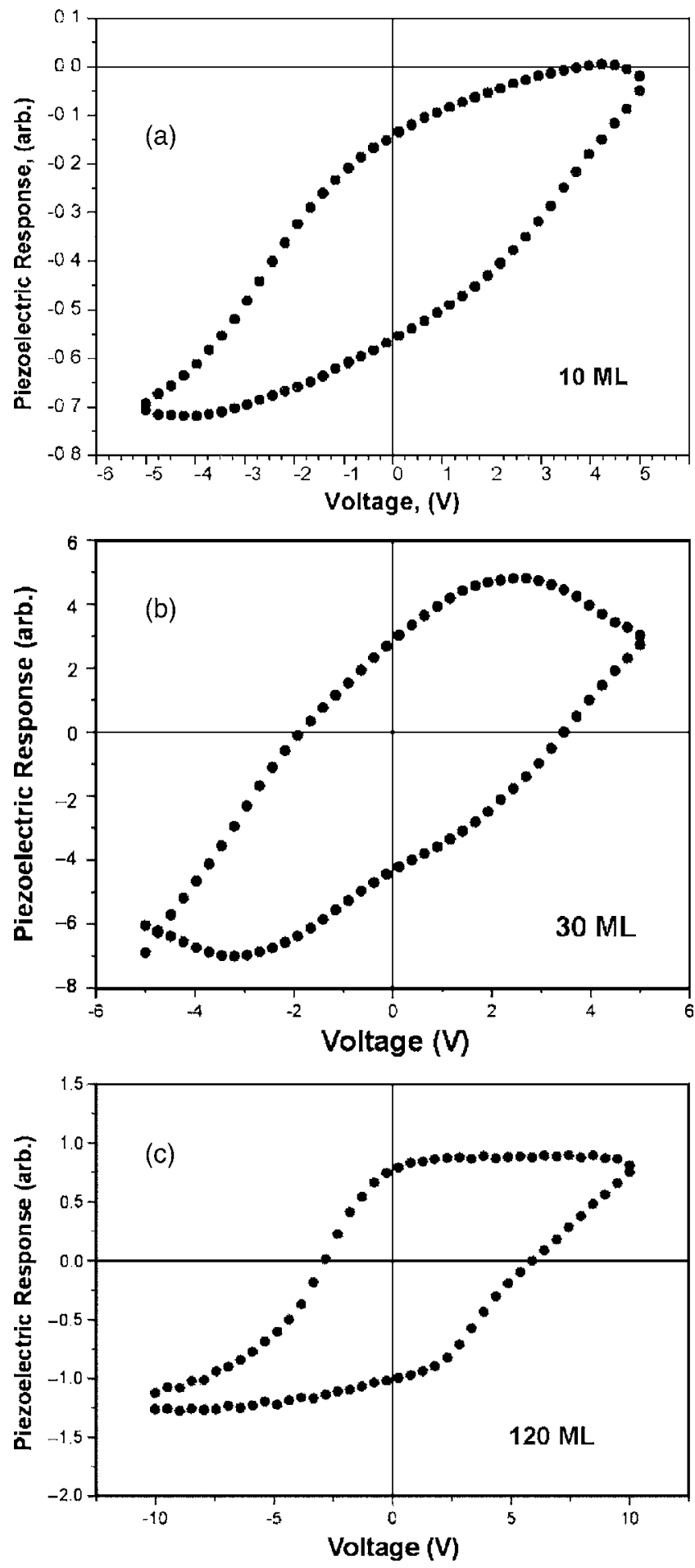

FIG. 1. Hysteresis loops for films (a) $10 \mathrm{ML}$, (b) $30 \mathrm{ML}$, (c) and $120 \mathrm{ML}$.

$V_{c}=2.7 \mathrm{~V}$. The hysteresis loop from the $120 \mathrm{ML}$ film [Fig. 1(c)] appears well saturated with a coercive voltage $V_{c}=4.4 \mathrm{~V}$.

The imaging of local switching was carried out by the method of point voltage pulse switching. In this method, the imaging area was first saturated by scanning the tip with a saturating bias of $-10 \mathrm{~V}$ and imaged under zero bias. Then, a small spot was switched by applying $+10 \mathrm{~V}$ to the tip at a specific location on the film. Figure 2 shows two views of the 30 ML film, which had the topography in Fig. 2(a). The PFM signal [amplitude $\times \sin$ (phase)] shown in Fig. 2(b) was recorded after application of a local voltage pulse of $+10 \mathrm{~V}$
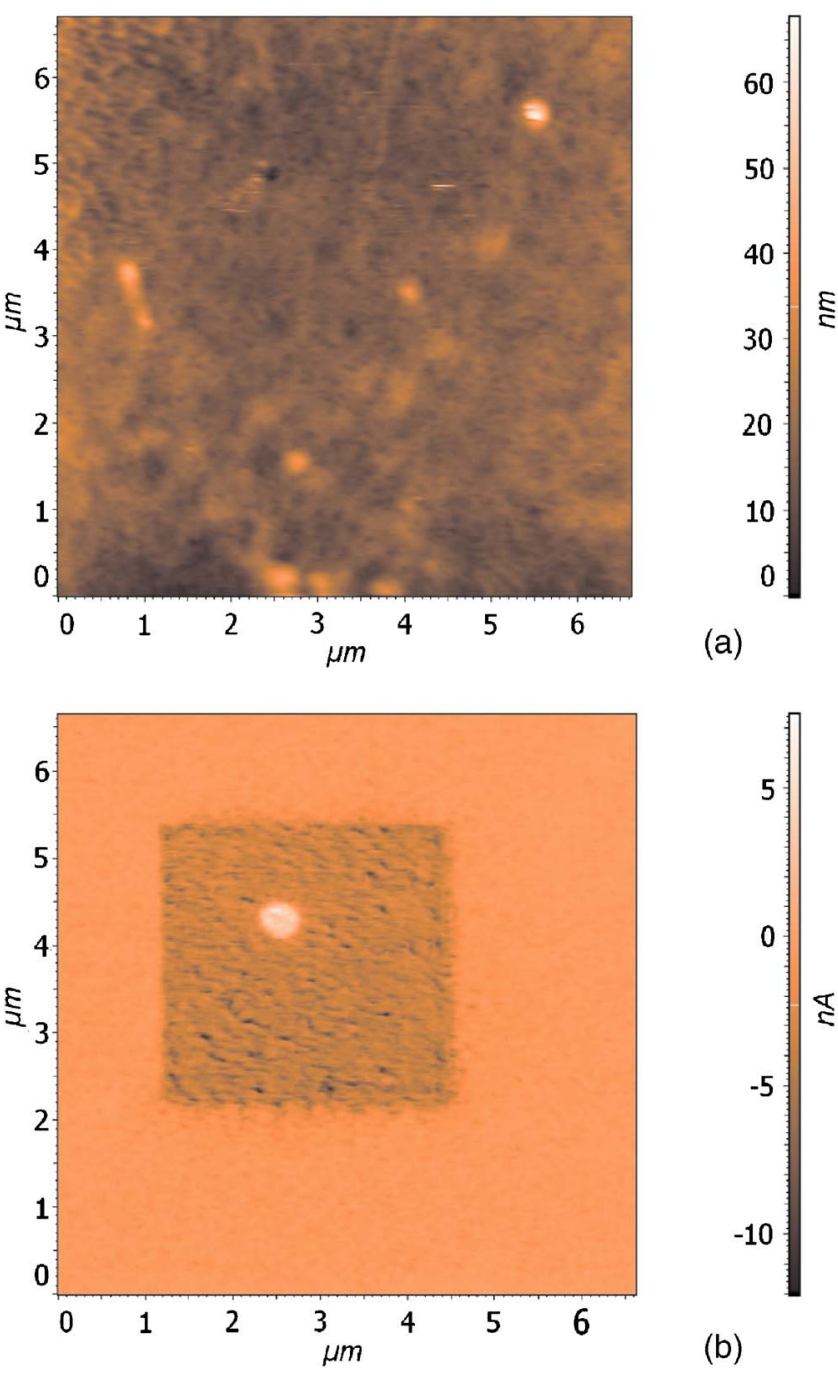

(a)

FIG. 2. (Color online) PFM images from the 30 ML film AFM topography image and (a) PFM topography and phase (b) image shows the square with $P=-P_{s}$ and spot with $P=+P_{s}$.

amplitude and $30 \mathrm{~s}$ duration shows a switched spot of approximately $500 \mathrm{~nm}$ in diameter.

The switching kinetics were studied by a series of local switching measurements. In this method, the imaging area was first saturated by scanning the tip with a saturating bias of $+10 \mathrm{~V}$ and imaged under zero bias. Then the local spot was switched, applying a voltage pulse of specified amplitude and duration at a fixed point and the resulting piezoresponse was imaged after applying the pulse. To determine the switching time for each voltage pulse amplitude, we recorded the piezoresponse after pulse application as a function of pulse duration and plotted this as function of pulse, as shown in inset to Fig. 3 for the 120 ML sample. The switching time was taken as the time at which this plot crosses zero piezoelectric response. In this way, the value of the switched piezoresponse was recorded as a function of voltage pulse amplitude and duration. A graph of switched piezoresponse versus pulse duration, for a given pulse amplitude, was then analyzed to determine the switching time $\tau$, defined at the time at which the piezoresponse crossed zero. ${ }^{9}$ Figure 3 shows the dependence of the switching time $\tau$ on pulse amplitude $V$ for the 10, 30 and 120 ML films.

The dependence of the switching time $\tau$ on switching voltage $V$ is a useful indicator of the dominant switching 


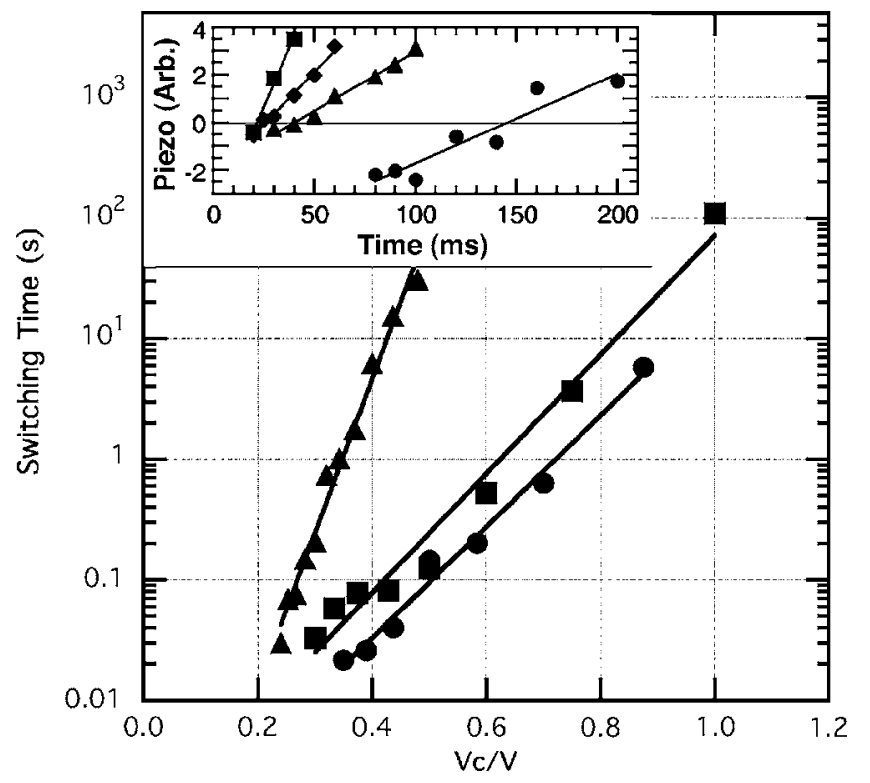

FIG. 3. Graph of $\log (t)$ vs $\left(V_{C} / V\right)$ for the $10 \mathrm{ML}$ film (purple triangles), the $30 \mathrm{ML}$ film, (green squares) and the $120 \mathrm{ML}$ film (blue circles). The solid lines are linear least squares fits to the data. Inset: peak PFM signal after switching from the $120 \mathrm{ML}$ sample as a function of switching pulse duration for pulse amplitudes of (left to right) 10, 9, 8, and $7 \mathrm{~V}$.

mechanism. The data in Fig. 3 are most consistent with nucleation-limited switching, which is an activation process with an exponential dependence on reciprocal voltage, expressed as follows: ${ }^{17}$

$$
(1 / \tau)=\left(1 / \tau_{0}\right) \exp \left(-a V_{C} / V\right),
$$

where $\tau_{0}$ is a constant and $a$ depends on reciprocal temperature. The fits in Fig. 3 return the following values for these parameters: $\tau_{0}=27 \times 10^{3} \mathrm{~s}$ and $a=29.3$ for the $10 \mathrm{ML}$ film, $\tau_{0}=1.2 \times 10^{3} \mathrm{~s}$ and $a=11.4$ for the $30 \mathrm{ML}$ film, and $\tau_{0}=2.1$ $\times 10^{3} \mathrm{~s}$ and $a=10.6$ for the $120 \mathrm{ML}$ film. Extrinsic switching shows no true threshold voltage, which appears to be the case here. If switching were domain-wall limited, the dependence would be a power law of the form ${ }^{17,18}(1 / \tau)=\left(1 / \tau_{0}\right)$ $\times\left(V / V_{C}\right)^{p}$, also with no true threshold. Intrinsic switching exhibits a true threshold with a square-root dependence of the form ${ }^{9}(1 / \tau)=\left(1 / \tau_{0}\right)\left(V / V_{C}-1\right)^{1 / 2}$, which form is clearly not consistent with the data in Fig. 3. The interpretation of the present experiments, however, is complicated by the fact that the applied electric field is far from uniform, decreasing as a function of distance from the tip. The switching voltage $V$ is not a single value, as required for the use of Eq. (1).

Previous studies have found that copolymer thin films made by solvent spinning ${ }^{19}$ and LB copolymer films of 30 ML or more ${ }^{9}$ exhibit extrinsic switching kinetics [Eq. (1)], while thinner films often ${ }^{6,9}$ (but not always ${ }^{10}$ ) exhibit intrin- sic switching kinetics. None of these studies, however, probed the effects of lateral dimensions on the switching mechanism. The present study of local switching kinetics measured using PFM supports the extrinsic nucleationinitiated switching mechanism in LB films of 10, 30 and 120 ML for a region approximately $500 \mathrm{~nm}$ in diameter. Further study of local switching kinetics in thin films and nanostructures is necessary if we are to understand the dominant mechanisms, and possibly control them.

The work in Moscow was supported the European INTAS program, Grant No. 1000008-8091, and by the Russian Foundation for Basic Research, Grant No. 05-02-16871. The work in Lincoln was supported by the National Science Foundation, Grant No. ECS-0600130.

${ }^{1}$ M. Okuyama and Y. Ishibashi, Ferroelectric Thin Films: Basic Properties and Device Physics for Memory Applications (Springer, Berlin, 2005).

${ }^{2}$ M. Alexe and A. Gruverman, Nanoscale Characterization of Ferroelectric Materials: Scanning Probe Microscopy Approach (Springer, Berlin, 2004).

${ }^{3}$ O. Kolosov, A. Gruverman, J. Hatano, K. Takahashi, and H. Tokumoto, Phys. Rev. Lett. 74, 4309 (1995).

${ }^{4}$ A. Gruverman, H. Tokumoto, A. S. Prakash, S. Aggarwal, B. Yang, M. Wuttig, R. Ramesh, O. Auciello, and T. Venkatesan, Appl. Phys. Lett. 71, 3492 (1999).

${ }^{5}$ S. Palto, L. Blinov, A. Bune, E. Dubovik, V. Fridkin, N. Petukhova, K. Verkhovskaya, and S. Yudin, Ferroelectr., Lett. Sect. 19, 65 (1995).

${ }^{6}$ A. Ievlev, K. Verkhovskaya, and V. Fridkin, Ferroelectr., Lett. Sect. 33, 147 (2006).

${ }^{7}$ A. V. Bune, V. M. Fridkin, S. Ducharme, L. M. Blinov, S. P. Palto, A. V. Sorokin, S. G. Yudin, and A. Zlatkin, Nature (London) 391, 874 (1998).

${ }^{8}$ S. Ducharme, V. M. Fridkin, A. V. Bune, S. P. Palto, L. M. Blinov, N. N. Petukhova, and S. G. Yudin, Phys. Rev. Lett. 84, 175 (2000).

${ }^{9}$ G. Vizdrik, S. Ducharme, V. M. Fridkin, and S. G. Yudin, Phys. Rev. B 68, 094113 (2003).

${ }^{10}$ H. Kliem and R. Tadros-Morgane, J. Phys. D: Appl. Phys. 38, 1860 (2005).

${ }^{11}$ R. Tadros-Morgane and H. Kliem, J. Phys. D 39, 4872 (2006); A. Tolstousov, R. V. Gaynutdinov, R. Tadros-Morgane, S. G. Yudin, A. L. Tolstikhina, H. Kliem, S. Ducharme, and V. M. Fridkin, Ferroelectrics 354, 99 (2007).

${ }^{12}$ H. Birk, J. Glatz-Reichenbach, Li-ie, E. Schreck, and K. Dransfeld, J. Vac. Sci. Technol. B 9, 1162 (1991); K. Noda, K. Ishida, A. Kubono, T. Horiuchi, H. Yamada, and K. Matsushige, Jpn. J. Appl. Phys., Part 1 40, 4361 (2001).

${ }^{13}$ L. M. Blinov, R. Barberi, S. P. Palto, M. P. De Santo, and S. G. Yudin, J. Appl. Phys. 89, 3960 (2001).

${ }^{14}$ B. J. Rodriguez, S. Jesse, S. V. Kalinin, J. Kim, and S. Ducharme, Appl. Phys. Lett. 90, 122904 (2007).

${ }^{15}$ S. Ducharme, S. P. Palto, and V. M. Fridkin, in Ferroelectric and Dielectric Thin Films, edited by H. S. Nalwa (Academic, San Diego, 2002), Vol. 3, pp. 545-591.

${ }^{16}$ M. Bai, A. V. Sorokin, D. W. Thompson, M. Poulsen, S. Ducharme, C. M. Herzinger, S. Palto, V. M. Fridkin, S. G. Yudin, V. E. Savchenko, and L. K. Gribova, J. Appl. Phys. 95, 3372 (2004).

${ }^{17}$ E. Fatuzzo and W. J. Merz, Phys. Rev. 116, 61 (1959).

${ }^{18}$ H. L. Stadler, J. Appl. Phys. 33, 3487 (1962).

${ }^{19}$ K. Kimura and H. Ohigashi, Jpn. J. Appl. Phys., Part 1 25, 383 (1986). 